\title{
Efecto del ácido lipoico sobre parámetros productivos y calidad de la canal en el pollo de engorda
}

\section{Effect of alpha-lipoic acid on productive parameters and carcass quality in broiler chickens}

\author{
Sergio Sigler Galvána, Sergio Gómez Rosalesb, Alma Delia Alarcón-Rojoc, \\ Lourdes Angelesb, Enrique Piñad, Armando Shimada Miyasakae, Ofelia Mora Izaguirree
}

\begin{abstract}
RESUMEN
En la producción de pollo de engorda se han empleado diferentes aditivos, entre ellos, los antioxidantes han tenido un papel relevante. Dentro de este tipo de productos el ácido alfa lipoico (AAL) ha destacado, pues se ha observado que su adición en la dieta ofrece diferentes beneficios a los animales. Por lo anterior, el objetivo del presente trabajo fue evaluar las respuestas productivas y la calidad de la carne del pollo de engorda con la administración de AAL en diferentes dosis $(0,40,80,160$ y 320 ppm). El experimento se dividió en dos fases, en la primera se evaluó el desempeño productivo de 1,000 aves que fueron divididas en 5 tratamientos con 200 animales cada uno, la duración de esta prueba fue $\mathbf{4 9}$ días. En la segunda fase se realizó el sacrificio de los animales, se obtuvieron 20 muestras por tratamiento y se realizaron pruebas de la calidad de la carne. Los resultados de las variables productivas y de las pruebas de calidad en la carne fueron analizados mediante un modelo completamente al azar. No se observaron diferencias significativas $(P>0.05)$ por la adición de AAL sobre las variables productivas; sin embargo, los pollos que recibieron $40 \mathrm{ppm}$ de AAL perdieron menor cantidad de agua por cocción.
\end{abstract}

PALABRAS Clave: Ácido alfa lipoico, Calidad de la carne, Pollo de engorda.

\begin{abstract}
Broilers production has been employed various additives, including antioxidants that have played an important role in it. Within these products, alpha lipoic acid (ALA) has distinguished; its addition in the diet provides different benefits to the animals. Therefore, the objective of this study was to evaluate the productive performance and meat quality of broilers with use of ALA in different doses $(0,40,80,160$ and $320 \mathrm{ppm})$. The experiment was divided into two phases, the first productive performance was evaluated using 1,000 birds that were divided into five treatments with 200 animals each; the duration of this test was $49 \mathrm{~d}$. In the second phase the sacrifice of animals was performed, 20 samples were obtained by treatment and testing the meat quality of them. The results of the production and quality meat variables were analyzed in a completely randomized design. No significant differences $(P>0.05)$ were found by the addition of ALA on productive variables; however, chicks receiving 40 ppm of ALA lost less water by cooking.
\end{abstract}

KEY WORDS: Alpha lipoic acid, Meat quality, Broilers.

En México, la producción de pollo ha mantenido una tendencia de crecimiento muy elevada,
Chicken production in Mexico has been increasing steadily in response to growing

\footnotetext{
Recibido el 6 de febrero de 2014. Aceptado el 8 de mayo de 2014.

a Programa de Posgrado en Ciencias de la Producción y de la Salud Animal, Universidad Nacional Autónoma de México (UNAM), México D.F. 04510, México.

b Centro Nacional de Investigación Disciplinaria en Fisiología y Mejoramiento Animal, INIFAP. Ajuchitlán, Colón, Qro. México.

c Facultad de Zootecnia y Ecología, Universidad Autónoma de Chihuahua. Chihuahua, Chih., México.

d Departamento de Bioquímica, Facultad de Medicina, UNAM. México D.F. México.

e Laboratorio de Rumiología y Metabolismo Nutricional (RuMeN), Secretaría de Posgrado, Facultad de Estudios Superiores-Cuautitlán, UNAM, Blvd. B. Quintana 514-D, Col. Arboledas, 76140, Querétaro, Qro. México. Tel 01442 2381032. ofemora66@unam.mx. Correspondencia al último autor.
} 
situación que ha sido influida principalmente por la creciente demanda de carnes blancas y en especial por sus precios, los cuales resultan altamente competitivos con respecto a otros cárnicos. Este elevado crecimiento de la producción, obedece a un fuerte nivel de tecnificación, similar al observado en países desarrollados, lo que se refleja en una alta eficiencia y en costos de producción bajos(1).

Durante los últimos 30 años, la selección avícola se centró en lograr aumentos en la velocidad de crecimiento de las líneas de carne; sin embargo, a consecuencia de esto se afectó negativamente el metabolismo del ave, su estructura muscular y la calidad de la carne(2). Además, se observó que las tasas elevadas de crecimiento pueden originar anormalidades morfológicas(2). Lo anterior, indica que las mejoras basadas en la selección genética tienen un límite que ya se está alcanzando, por ello los avicultores han optado por el uso de aditivos para mejorar la producción, tales como coccidiostatos, enzimas, saborizantes, antioxidantes, por mencionar algunos. Dentro de los antioxidantes se encuentra el ácido alfa lipoico ( $A A L)$, que es un ácido graso derivado del ácido octanóico que integra en su estructura dos átomos de azufre, los cuales le confieren varias de sus características particulares. El AAL participa como cofactor de dos complejos enzimáticos importantes en el ciclo de Krebs: la piruvato deshidrogenasa y la alfacetoglutarato deshidrogenasa(3).

El AAL ha sido usado en animales, tanto de laboratorio $(4,5)$ como de granja (pollo de engorda, codornices y cerdos), en los que se ha observado una mejora en la ganancia de peso y la disminución de problemas asociados con el estrés calórico y el síndrome ascítico, además del papel que ha desempeñado en la estimulación de la sensibilidad a la insulina(610). El Cuadro 1 muestra algunos experimentos en los que el AAL ha sido evaluado empleando distinto modelo animal, cuyos resultados revelan efectos positivos de ese ácido sobre la ganancia de peso y en diferentes variables de calidad en la carne. Sin embargo, los modelos experimentales demand for white meats, and its more competitive pricing compared to other meats. This high growth rate has been facilitated by production technology analogous to that in developed countries, high efficiency rates and low production costs(1).

Genetic selection in poultry during the last thirty years has focused on augmenting growth rates in broiler lines. However, this has negatively affected animal metabolism, muscle structure and meat quality(2). Very high growth rates can also cause morphological abnormalities(2). Apparently, line improvement based on genetic selection is reaching its limit. To improve production, poultry producers are now opting for use of additives such as coccidiostats, enzymes, flavorings and antioxidants, among others. Alpha-lipoic acid (ALA) is a fatty acid derived from octanoic acid. It includes two sulphur atoms in its structure, which confer it specific characteristics. It participates as a cofactor in two enzymatic complexes in the Krebs cycle: pyruvate dehydrogenase and $\alpha$-ketoglutarate dehydrogenase(3).

As an antioxidant, ALA has been used in laboratory animals $(4,5)$, as well as farm animal systems (e.g. broiler chickens, quail and pigs). Addition of ALA has been shown to improve weight gain, decrease problems associated with caloric stress and ascites syndrome, and stimulate insulin sensitivity(6-10). Although many studies have shown ALA's positive effects on weight gain and meat quality (Table 1 ), very different experimental models and inclusion levels (e.g. 10 to 10,000 ppm) were used, which may partially explain the wide variation in results.

The present study objective was to test five ALA inclusion levels (0, 40, 80, 160 and 320 ppm) in broiler chicken diets and evaluate their effects on production parameters and meat quality.

Animal trials were run at the Ajuchitlán, Querétaro, experimental farm of the National Center for Disciplinary Research in Animal 
y las dosis utilizadas han sido muy amplias (de 10 a 10,000 ppm), lo que podría explicar parte de la variación que se observa en los resultados obtenidos.

Tomando como referencia las diversas dosis administradas (Cuadro 1), se optó por emplear como base el trabajo de $\operatorname{Díaz}(8)$, donde se usaron 10, 20 y 40 ppm, presentando el mejor resultado la dosis de $40 \mathrm{ppm}$. No obstante esto, se quería saber si con dosis más altas mejoraría la ganancia de peso y la calidad de la carne, por lo que se decidió usar además 80,160 y $320 \mathrm{ppm}$ de AAL.

Por lo anterior, el objetivo de este trabajo fue probar tales dosis de AAL $(0,40,80,160$ y 320 ppm) incorporadas en la dieta de pollos de engorda para evaluar su efecto en las variables productivas y en la calidad de la carne.

La investigación se realizó en la granja experimental del Centro Nacional de Investigación Disciplinaria en Fisiología y Mejoramiento Animal (CENID-FyMA), del Instituto Nacional de Investigaciones Forestales, Agrícolas y Pecuarias (INIFAP), ubicado en Ajuchitlán, Querétaro. Las pruebas de calidad de carne se realizaron en el laboratorio de
Physiology and Improvement (Centro Nacional de Investigación Disciplinaria en Fisiología y Mejoramiento Animal - CENID-FyMA) of the National Institute for Forestry, Agriculture and Livestock Research (Instituto Nacional de Investigaciones Forestales, Agrícolas y Pecuarias - INIFAP). Meat quality tests were done at the Biochemistry Laboratory of the Faculty of Zootechny and Ecology, Autonomous University of Chihuahua (Universidad Autónoma de Chihuahua).

Trials were run using a total of 1,000 recently hatched male Ross 308 line chicks. Two hundred chicks were assigned to each treatment (i.e. inclusion level), and divided into eight corrals per treatment (each corral was a replicate), with 25 animals per corral. Corrals were distributed using a random block design. Previous studies had shown wide variation in results due to drastic differences in ALA inclusion levels. One study using 10, 20 and $40 \mathrm{ppm}$ levels in broiler chickens showed optimum results at the $40 \mathrm{ppm}$ level( 8$)$. However, slightly higher inclusion levels may improve weight gain and meat quality. Five dietary ALA levels ( 0 , $40,80,160$ and $320 \mathrm{ppm}$ ) were tested throughout the three-phase, 49-d experimental

Cuadro 1. Experimentos en los cuales se usó el ácido lipoico sobre distinto modelo animal

Table 1. Some previous studies using lipoic acid in animal models

\begin{tabular}{|c|c|c|c|}
\hline Authors & Concentration & Model & Effect \\
\hline Hamano et al, 1999(34) & 5 and $50 \mathrm{ppm}$ & Broiler chickens & $\begin{array}{l}\text { Collaborates in energy metabolism as possible facilitator in } \\
\beta \text {-adrenergic response }\end{array}$ \\
\hline Berg et al, 2003(7) & 150 ppm & Finished pigs & Improved loin pH \\
\hline Díaz et al, 2003(8) & 10,20 and $40 \mathrm{ppm}$ & Broiler chickens & $\begin{array}{l}40 \mathrm{ppm} \text { improved feed conversion and lowered mortality from } \\
\text { ascites }\end{array}$ \\
\hline Shen et al, 2005(4) & 5,000 and $10,000 \mathrm{ppm}$ & Mice & $\begin{array}{l}\text { Increased muscle tissue, reduced adipose tissue and raised } \\
\text { carcass } \mathrm{pH}\end{array}$ \\
\hline Hamano, 2006(10) & 200 and $400 \mathrm{ppm}$ & Broiler chickens & Stimulated tissue insulin sensitivity and promoted lipolysis \\
\hline Wang et al, 2010(5) & $0.75 \%$ in drinking water & Senile mice & $\begin{array}{l}\text { Improved carcass composition and lean mass, promoted } \\
\text { mitochondrial biogenesis }\end{array}$ \\
\hline Arshad et al, 2011(6) & $\begin{array}{l}25,75 \text { and } 150 \mathrm{ppm}+ \\
\alpha \text {-tocopherol }\end{array}$ & Broiler chickens & Promoted better carcass shelf life \\
\hline Halici et al, 2011(9) & $250 \mathrm{ppm}$ & Quail & Counteracted effects of caloric stress \\
\hline
\end{tabular}


Bioquímica de la Facultad de Zootecnia y Ecología de la Universidad Autónoma de Chihuahua.

Se empleó un total de 1,000 pollitos machos, recién eclosionados, de la línea Ross 308, usando 200 animales por tratamiento, los cuales se dividieron en ocho corrales para cada dosis probada, colocando 25 animales por corral, cada corral correspondió a una repetición. Los corrales se distribuyeron mediante un diseño de bloques al azar. Se probaron cinco niveles de AAL: 0 , $40,80,160$ y $320 \mathrm{ppm}$ en el alimento, que fueron adicionados durante toda la engorda (49 días). Se evaluó la ganancia diaria de peso, el consumo de alimento, la conversión alimenticia $y$ el peso final.

Los animales se alimentaron con un producto

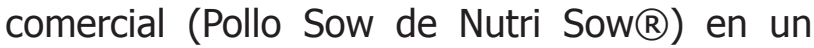
esquema de tres fases, cada una con las características que se indican en el Cuadro 2. El alimento comercial se molió y fue colocado en una mezcladora donde se adicionaron las diferentes dosis de AAL; después el alimento mezclado se identificó y almacenó para proporcionárselo a las aves posteriormente.

Los animales se mantuvieron en un horario de $24 \mathrm{~h}$ de luz durante todo el experimento, a fin de promover un mayor consumo de alimento. No se aplicó ninguna vacuna ni se adicionó otro aditivo ajeno al alimento, ya que éste incluía vitaminas y minerales, además, en las fases II y III se incluían pigmentos amarillos.

Al terminar la Fase III todos los pollos se sacrificaron en la planta de procesamiento de aves Tipo Inspección Federal Pollo de Querétaro S. A. de C. V., localizada en el municipio de Colón, Querétaro. Previamente al sacrificio, los animales se ayunaron durante un periodo de $10 \mathrm{~h}$, permitiéndoseles sólo el acceso al agua ad libitum. Al momento del sacrificio se seleccionaron, al azar, 20 canales por cada tratamiento (incluyendo todas las repeticiones), las cuales se enfriaron a $4^{\circ} \mathrm{C}$ durante $24 \mathrm{~h}$. Posteriormente, se tomaron las muestras que period. Data were collected on daily weight gain, feed intake, feed conversion rate and final weight.

A commercial feed was administered in a threephase growth scheme (Table 2). The commercial feed was ground, placed in a mixer and ALA added in the amount required to produce diets containing the five tested ALA proportions. Prepared diets were identified and stored until use.

A $24 \mathrm{~h}$ light photoperiod was used throughout the experimental period to promote greater feed intake. No vaccines were administered to animals. Neither were other additives included in the diets since the commercial feed was formulated with vitamins and minerals. Yellow pigments were added in phases II and III.

At the end of Phase III all animals were slaughtered at a Federal Inspection Type (Tipo Inspección Federal - TIF) poultry processing plant (Pollo de Querétaro S.A. de C.V., Colón, Querétaro). Before they were killed the animals were fasted for $10 \mathrm{~h}$, with free access to water. When killed, 20 carcasses were selected randomly from each treatment (including all replicates), and cooled to $4^{\circ} \mathrm{C}$ for $24 \mathrm{~h}$. Two samples of breast meat were then taken, one to measure cooking loss and the other for the remaining tests. The breasts were frozen at $-30{ }^{\circ} \mathrm{C}$ until use.

Cuadro 2. Composición proximal del alimento empleado en las fases de producción de pollo de engorda (\%)

Table 2. Proximate composition of feed used in three broiler chicken production phases (\%)

\begin{tabular}{lccc}
\hline Composition & $\begin{array}{c}\text { Phase 1 } \\
(1-21 \mathrm{~d})\end{array}$ & $\begin{array}{c}\text { Phase II } \\
(22-35 \mathrm{~d})\end{array}$ & $\begin{array}{c}\text { Phase III } \\
(36-49 \mathrm{~d})\end{array}$ \\
\hline Moisture & 12.0 & 12.0 & 12.0 \\
Fat & 3.0 & 3.0 & 3.0 \\
NFE & 54.0 & 56.5 & 59.5 \\
Protein & 21.5 & 19.5 & 17.5 \\
Crude fiber & 3.5 & 3.5 & 3.0 \\
Ash & 6.0 & 5.5 & 5.0 \\
\hline
\end{tabular}


serían usadas para las pruebas de calidad de la carne, obteniendo dos muestras de pechuga por cada canal, una sería usada para medición de la pérdida de agua por cocción y la otra sería empleada en el resto de las pruebas. Las pechugas se congelaron a $-30^{\circ} \mathrm{C}$ hasta su uso.

Para determinar la calidad de la carne se realizaron las siguientes pruebas:

Rendimiento de la pechuga. Después del sacrificio las canales se procesaron de forma convencional, almacenándose a $4{ }^{\circ} \mathrm{C}$ durante $24 \mathrm{~h}$. Pasado este periodo se tomaron los pesos de las canales en frío y, posteriormente, el peso de las pechugas deshuesadas, considerando tanto el músculo pectoral mayor como el menor, expresando el resultado como porcentaje del peso de la canal en frío.

Determinación del pH. El pH de las carne se midió durante dos ocasiones en el músculo pectoral o pechuga (Pectoralis major), con un potenciómetro portátil de la marca HANNA modelo HI 99163 (HANNA® instruments, USA). La primera medición del $\mathrm{pH}$ se tomó a los 15 min post mortem $(11,12)\left(\mathrm{pH}_{15}\right)$ insertando el electrodo a $1 \mathrm{~cm}$ de profundidad, con calibraciones del equipo cada 30 a 50 mediciones con soluciones buffer de $\mathrm{pH}=4 \mathrm{y}$ $\mathrm{pH}=7(12,13)$. Las canales se enfriaron durante $24 \mathrm{~h}$ para alcanzar una temperatura interna de $4{ }^{\circ} \mathrm{C}$; posteriormente, se procedió a medir el $\mathrm{pH}$ final a las $24 \mathrm{~h}$ post mortem $\left(\mathrm{pH}_{24}\right)$, justo en el mismo punto en donde se realizó la lectura anterior $\mathrm{pH}_{15}{ }^{(12)}$.

Determinación del color. Se usó un colorímetro modelo CR-410 (Konica Minolta, USA) el cual tiene como referencia el sistema CIE L*a*b*(14), determinando los valores de luminosidad $\left(L^{*}\right)$, índice de rojo (a*) e índice de amarillo (b*). El iluminante seleccionado fue D65, con un ángulo de medición de $2^{\circ}$ y enfoque de $8 \mathrm{~mm}(12,15)$. La medición del color se realizó a las 24 h post mortem, tomándose la lectura en la zona de la pechuga, tanto sobre la piel como sobre el músculo pectoral mayor. Para el caso del
Six meat quality variables were measured using the breast meat:

Breast yield. The carcasses were processed using conventional techniques and stored at 4 ${ }^{\circ} \mathrm{C}$ for $24 \mathrm{~h}$. They were then weighed cold, and again after deboning, considering both the pectoralis major and minor muscles. Results were expressed as a percentage of cold carcass weight.

$\mathrm{pH}$. Meat $\mathrm{pH}$ was measured twice in the pectoralis major or breast, using a portable potentiometer (HI 99163, HANNA ${ }^{\circledR}$ Instruments, USA). The first measurement was taken $15 \mathrm{~min}$ post mortem $\left(\mathrm{pH}_{15}\right)$ by inserting the electrode to $1 \mathrm{~cm}$ depth and calibrating the device every 30 to 50 measurements using $\mathrm{pH} 4$ and $\mathrm{pH} 7$ buffer solutions $(11,12,13)$. After chilling for $24 \mathrm{~h}$ to lower breast internal temperature to $4{ }^{\circ} \mathrm{C}$, a second measurement was taken $\left(\mathrm{pH}_{24}\right)$ at exactly the same point at which $\mathrm{pH}_{15}$ had been measured(12).

Color. Based on the CIE L*a*b* system(14), luminosity $\left(\mathrm{L}^{*}\right)$, red index $\left(\mathrm{a}^{*}\right)$ and yellow index (b*) values were measured using a colorimeter (CR-410, Konica Minolta, USA). The selected illuminant was DE65, with a $2^{\circ}$ measurement angle and an $8 \mathrm{~mm}$ focus $(12,15)$. Color measurements were taken $24 \mathrm{~h}$ post mortem on the breast, in areas of skin and muscle tissue. To measure color on the muscle tissue, while avoiding the excessive shine found on the breast muscle surface, the skin was removed and a measurement taken on the medial surface, wishbone side $(11,12,16)$.

The deboned breasts were stored in a vacuum and frozen at $-30{ }^{\circ} \mathrm{C}$ until further use. Before measuring other variables, the breasts were defrosted at $4{ }^{\circ} \mathrm{C}$ overnight, then left at room temperature until fully defrosted.

Water holding capacity. Following the technique of Grau and Hamm(17), a $0.3 \mathrm{~g}$ sample was placed between two sheets of $0.5 \mu$ porosity filter paper, and then between two $12 \times 12 \mathrm{~cm}$ 
músculo, antes de la medición se retiró la piel y se midió el color sobre la superficie medial del lado de la fúrcula con el fin de evitar el brillo excesivo de la superficie del músculo de la pechuga $(11,12,16)$.

Posteriormente, las pechugas deshuesadas fueron envasadas al vacío y congeladas a -30 ${ }^{\circ} \mathrm{C}$ hasta el momento de efectuar las demás mediciones. Para descongelar las pechugas, éstas se colocaron en refrigeración a $4^{\circ} \mathrm{C}$ durante toda la noche, en la mañana siguiente se retiraron del refrigerador y se mantuvieron a temperatura ambiente para finalizar el proceso de descongelación.

Capacidad de retención de agua. Se midió con la técnica de Grau y Hamm(17) modificada, en la cual se utilizó una muestra de $0.3 \mathrm{~g}$ que fue colocada entre dos hojas de papel filtro con porosidad de $0.5 \mu$, para después ser posicionada entre dos placas de plexiglass de $12 \times 12 \mathrm{~cm}^{2}$ aplicando una fuerza con un peso de $10 \mathrm{~kg}$ durante $15 \mathrm{~min}(17,18)$. El porcentaje de agua perdida se expresó como agua libre y la capacidad de retención de agua se determinó restando el agua libre de 100.

Pérdida por goteo. Se determinó por el método gravimétrico a las $24 \mathrm{~h}$ post mortem, usando una porción del músculo pectoral de $3 \mathrm{~g}$ que fue suspendida dentro de un recipiente de plástico lo más hermético posible, a fin de evitar que la muestra se secara, manteniéndose a 3 ${ }^{\circ} \mathrm{C}$ durante $48 \mathrm{~h}$. El resultado se expresó como porcentaje de la masa muscular inicial, esto es [peso perdido por goteo/peso inicial de la carne] x $100(12,16,19)$.

Agua perdida por cocción. Para este análisis, la carne se empaquetó al vacío y se congeló a -30 ${ }^{\circ} \mathrm{C}$, posteriormente la carne (músculo Pectoralis major) se descongeló como se explicó previamente, para después pesarla y cocerla en agua a $85^{\circ} \mathrm{C}$ durante 15 min, hasta alcanzar una temperatura interna de 75 a $80{ }^{\circ} \mathrm{C}$, se enfrió y pesó nuevamente, obteniendo así el agua perdida por descongelación-cocción; el
Plexiglas squares under $10 \mathrm{~kg}$ of force for 15 $\min (17,18)$. Water loss was expressed as released water, and water holding capacity (WHC) calculated by subtracting free water values from 100.

Drip-loss. Twenty-four hours post mortem, a $3 \mathrm{~g}$ portion of breast muscle was suspended inside a hermitically sealed plastic container (to avoid water loss from evaporation) for $48 \mathrm{~h}$ at $3^{\circ} \mathrm{C}$. Results were expressed as a percentage of initial muscle mass: [weight loss by drip/initial weight] x $100(12,16,19)$.

Cooking loss. Breast meat was packed in a vacuum, frozen at $-30{ }^{\circ} \mathrm{C}$ and defrosted as described previously. It was then weighed, and cooked in water at $85{ }^{\circ} \mathrm{C}$ for $15 \mathrm{~min}$, until reaching 75 to $80{ }^{\circ} \mathrm{C}$ internal temperature. It was cooled and weighed again. Loss from freezing/cooking was expressed as a percentage of fresh muscle weight(11).

Texture. This variable was quantified in cooked muscle tissue by measuring shear force (WarnerBratzler) with a materials tester (LS1, Lloyd Instruments, USA). Cooking technique (i.e. heating rate and final temperature in thermal center) was standardized, and internal temperature varied from 75 to $80{ }^{\circ} \mathrm{C}$. Sample size, location within the muscle, fiber orientation vis-a-vis the cutting blade and presence of connective tissue were controlled for(12). A minimum of three samples $(1 \mathrm{~cm}$ high $\times 1.5 \mathrm{~cm}$ wide $\times 5 \mathrm{~cm}$ long) from each breast sample were cut parallel to the longitudinal orientation of the muscle fibers. During cutting, samples were positioned with muscle fibers perpendicular to the blade(12).

Productive variable and meat quality data were analyzed with a completely random model, using the general linear models procedure. Differences between the means were identified with the least squares differences method. A distribution test was run for the $\mathrm{pH}$ data, and all the percentage values were arc-sine square-root transformed for analysis. 
resultado obtenido se expresó como porcentaje del peso del músculo fresco(11).

Textura. La textura se evaluó midiendo la fuerza de corte (Warner-Bratzler) con el equipo LLOYD LS1 (USA), empleando cortes de carne después de cocinarse, por lo que se estandarizó el método de cocinado (velocidad de calentamiento y temperatura final en el centro térmico). La temperatura interna osciló entre 75 y $80^{\circ} \mathrm{C}$. Siempre se tuvo en cuenta el tamaño de muestra, la localización dentro del músculo, la orientación de las fibras a la hoja de la cuchilla y la presencia de tejido conectivo(12). Las muestras se cortaron paralelas a la orientación longitudinal de las fibras musculares tomando siempre una muestra del mismo tamaño $(1 \mathrm{~cm}$ de alto $\times 1.5 \mathrm{~cm}$ de ancho $\times 5 \mathrm{~cm}$ de largo), y se repitió tres veces para cada muestra de pechuga como mínimo. Las muestras se posicionaron de forma que la navaja cortara las fibras de manera perpendicular(12).

Los datos de las variables productivas y los obtenidos de las pruebas para calidad de la carne se analizaron mediante un modelo completamente al azar, con el procedimiento de modelos lineales generales. Las diferencias entre medias se evaluaron usando el método de las diferencias mínimo cuadráticas. Se realizó una prueba de distribución para el caso del pH,
No differences in final weight and weight gain were identified between the 40, 80 and 160 ppm treatments. The $320 \mathrm{ppm}$ treatment had values below the other ALA treatments but not different $(P>0.05)$ from the control treatment (i.e. $0 \mathrm{ppm})($ Table 3$)$. Feed intake was lower $(P<0.05)$ in the $320 \mathrm{ppm}$ treatment than in all other treatments, including the control. Feed conversion rate (FCR) differed between treatments; the 80, 160 and $320 \mathrm{ppm}$ treatments had values lower $(P<0.05)$ than those of the 0 and $40 \mathrm{ppm}$ treatments. The present results partially coincide with a study of the effects of ALA in pig diets in which no effects were observed on carcass weight(7).

The present data for total feed intake, final weight and weight gain showed that the 320 ppm ALA level had a negative effect on these variables. This may be caused by a decrease in hypothalamic AMPK activity in response to this inclusion level. Voluntary feed intake would consequently decrease, leading to lower weight gain and lower carcass weight, as reported elsewhere(20). The present results support those of studies in which the lowest weights were observed at the highest ALA inclusion level $(150 \mathrm{mg} / \mathrm{kg}$ feed) administered in tandem with $\alpha$-tocopherol (200 ATA mg/kg feed). In another study, addition of $40 \mathrm{ppm}$ ALA in broiler chickens had no effect on feed intake, but did increase

Cuadro 3. Efecto de diferentes niveles de ácido alfa lipoico (ALA) en la dietas sobre parámetros productivos de pollos de engorda

Table 3. Effect of different $\alpha$-lipoic acid (ALA) inclusion levels on production parameters in broiler chickens

\begin{tabular}{|c|c|c|c|c|c|c|c|c|}
\hline \multirow[b]{2}{*}{ ALA (ppm) } & \multicolumn{2}{|c|}{ Final weight $(\mathrm{kg})$} & \multicolumn{2}{|c|}{ Total intake (kg) } & \multicolumn{2}{|c|}{ Weight gain $(\mathrm{kg})$} & \multicolumn{2}{|c|}{ Feed conversion } \\
\hline & $\bar{X}$ & SE & $\bar{X}$ & SE & $\bar{X}$ & SE & $\bar{X}$ & SE \\
\hline 0 & $2.24^{a, b}$ & 0.02 & $5.06^{a}$ & 0.06 & $2.19^{a, b}$ & 0.02 & $2.26^{a}$ & 0.02 \\
\hline 40 & $2.27^{a}$ & 0.02 & $5.18^{a}$ & 0.06 & $2.23^{a}$ & 0.02 & $2.28^{a}$ & 0.02 \\
\hline 80 & $2.32^{a}$ & 0.02 & $5.17^{a}$ & 0.06 & $2.28^{a}$ & 0.02 & $2.23 \mathrm{ab}$ & 0.02 \\
\hline 160 & $2.30^{a}$ & 0.02 & $5.06^{a}$ & 0.06 & $2.26^{a}$ & 0.02 & $2.19^{b}$ & 0.02 \\
\hline 320 & $2.18^{b}$ & 0.02 & $4.86^{b}$ & 0.06 & $2.14^{\mathrm{b}}$ & 0.2 & $2.23 \mathrm{ab}$ & 0.02 \\
\hline
\end{tabular}

a,b Different letter superscripts in the same column indicate significant difference $(P<0.05), \mathrm{n}=20$.

$\mathrm{SE}=$ Standard error. 
y todos los valores que estaban expresados como porcentaje se transformaron a la raíz cuadrada del arco-seno para su análisis.

En el Cuadro 3 se observan las medias y el error estándar del peso final, ganancia de peso, consumo de alimento y conversión alimenticia en 49 días de evaluación. Respecto al peso final y la ganancia de peso, se puede observar que no existió diferencia entre los tratamientos 40, 80 y 160 ppm, sólo el nivel de 320 ppm de AAL mostró valores menores que el resto de las dosis, pero iguales al testigo $(P>0.05)$. A su vez, el consumo de alimento fue menor $(P<0.05)$ en los animales que recibían 320 ppm de AAL con respecto a todos los tratamientos incluyendo al testigo. En el caso de la conversión alimenticia se observaron algunas diferencias entre tratamientos, la dosis de 160 ppm presentó el menor valor, siendo diferente a los niveles de 0 y $40 \mathrm{ppm}$, pero igual al resto de los tratamientos $(P<0.05)$. En los experimentos realizados por Berg et a( $(7)$ con cerdos, no se observaron efectos sobre el peso de la canal al adicionar ácido alfa lipoico en su dieta.

Como se puede ver en los resultados de consumo total de alimento, peso final y ganancia de peso del presente trabajo, la mayor dosis de AAL (320 ppm) ejerció un efecto negativo daily weight gain and feed conversion(8). However, other authors report no differences in chickens fed different ALA levels(22).

Meat color exhibited lower $L^{*}$ values for all treatments except the $320 \mathrm{ppm}$, a lower a* value in the $160 \mathrm{ppm}$ treatment, and lower $\mathrm{b}^{*}$ values in all the ALA treatments (Table 4). Skin color did not differ $(P>0.05)$ between treatments. No data have been published on color measurements directly on breast meat from chickens fed ALA-supplemented diets, although color data have been reported for raw chicken meat in the form of nuggets and hamburgers(21). In the nuggets at day 0 , the control did not differ from the lowest ALA level (25 mg ALA/kg feed), which, in turn, did not differ from the other tested ALA levels. The hamburger patties in the control also did not differ from the lowest level, but did differ from the treatments with higher ALA levels. In the present results, the breast meat exhibited higher yellow ( $\left.b^{*}\right)$ than red $\left(a^{*}\right)$ values. This could be partially due to low final $\mathrm{pH}$, which reduces myoglobin's absorption of red light (negative a* value), resulting in meat that appears less red and more yellow(23).

Only the color of breast meat was evaluated here. It should be noted that breast meat has

Cuadro 4. Efecto de diferentes niveles de ácido alfa lipoico (ALA) en la dietas sobre el color de la carne y piel de pollos de engorda

Table 4. Effect of different $\alpha$-lipoic acid (ALA) inclusion levels on meat and skin color in broiler chickens

\begin{tabular}{|c|c|c|c|c|c|c|c|c|c|c|c|c|}
\hline \multirow[b]{3}{*}{ ALA (ppm) } & \multicolumn{6}{|c|}{ Meat } & \multicolumn{6}{|c|}{ Skin } \\
\hline & \multicolumn{2}{|l|}{$L^{*}$} & \multicolumn{2}{|l|}{$a^{*}$} & \multicolumn{2}{|l|}{$b^{*}$} & \multicolumn{2}{|l|}{$\mathrm{L}^{*}$} & \multicolumn{2}{|l|}{$a^{*}$} & \multicolumn{2}{|l|}{$b^{*}$} \\
\hline & $\bar{X}$ & SE & $\bar{x}$ & SE & $\bar{X}$ & $\overline{S E}$ & $\bar{x}$ & $\overline{S E}$ & $\bar{X}$ & SE & $\bar{x}$ & SE \\
\hline 0 & $55.18^{b}$ & 0.49 & $11.45^{a}$ & 0.58 & $23.24^{b}$ & 0.46 & 72.46 & 1.06 & 7.18 & 0.54 & 38.45 & 1.49 \\
\hline 40 & $53.11^{\mathrm{a}}$ & 0.48 & $9.88 \mathrm{a}, \mathrm{b}$ & 0.57 & $21.61^{a}$ & 0.46 & 72.21 & 1.06 & 7.21 & 0.54 & 35.49 & 1.49 \\
\hline 80 & $52.70^{a}$ & 0.48 & $10.07^{\mathrm{a}}$ & 0.57 & $22.43^{a, b}$ & 0.46 & 71.97 & 1.12 & 7.51 & 0.57 & 39.44 & 1.57 \\
\hline 160 & $52.25^{a}$ & 0.48 & $8.44^{b}$ & 0.57 & $22.13^{a, b}$ & 0.46 & 71.75 & 1.12 & 8.07 & 0.57 & 35.97 & 1.57 \\
\hline 320 & $53.86^{a, b}$ & 0.48 & $10.30^{a}$ & 0.57 & $21.80^{a}$ & 0.46 & 73.24 & 1.06 & 6.98 & 0.54 & 36.32 & 1.49 \\
\hline
\end{tabular}

$L^{*}=$ Luminosit; $a^{*}=$ Red index; $b^{*}=$ Yellow index.

a,b Different letter superscripts in the same column indicate significant difference $(P<0.05), \mathrm{n}=20$.

SE $=$ Standard error. 
debido a que en estos parámetros se obtuvieron los valores más bajos, esto puede deberse a que dicha dosis disminuye la actividad de la AMPK hipotalámica, reduciendo de esta forma, el consumo voluntario, lo que trae como consecuencia menor ganancia de peso y por ende menor peso corporal, tal como se ha visto en trabajos previos(20).

Los resultados obtenidos con la mayor dosis, $320 \mathrm{ppm}$, concuerdan con lo observado por otros autores $(6,21)$, quienes administraron ácido alfa lipoico junto con alfa-tocoferol y observaron que el grupo con la mayor dosis (150 mg AAL + $200 \mathrm{mg} \mathrm{ATA} / \mathrm{kg}$ de alimento) presentó el menor peso. En este sentido los resultados de Díaz et a(8) muestran que $40 \mathrm{ppm}$ de AAL en la dieta, no tuvieron efecto sobre el consumo de alimento, pero sí mejoraron la ganancia diaria de peso y la conversión alimenticia. Sin embargo, otros autores(22) no encontraron diferencias entre sus tratamientos con diferentes dosis en los pollos de engorda.

En el Cuadro 4 se aprecia que en el caso del color de la carne, hubo una disminución de $L^{*}$ para todos los tratamientos que recibieron AAL excepto $320 \mathrm{ppm}$, un menor valor de a* para la dosis de 160 ppm y una disminución de $b^{*}$ para todos los niveles de AAL. En el caso del color de la piel, no hubo diferencias $(P>0.05)$. Respecto a los valores de color de la carne, a lower myoglobin concentration, and therefore a lighter color, than other pieces such as the leg(24). All the tested ALA levels affected $(P<0.05)$ meat color. Compared to the control, the 40 and $160 \mathrm{ppm}$ levels resulted in less luminous meat with less red and yellow intensity. The $80 \mathrm{ppm}$ treatment produced meat that was less luminous, but with the same red and yellow intensities as the control. Finally, the $320 \mathrm{ppm}$ treatment only lowered yellow intensity, having no effect on luminosity or red intensity. In contrast, none of the treatments affected skin color.

Of the ALA treatments, the $160 \mathrm{ppm}$ had the highest $(P<0.05)$ shear force value, meaning that addition of ALA generally lowered meat tenderness versus the control (Table 5). This differs from a study reporting the effect of ALA inclusion at $0,30,60$ and $90 \mathrm{~d}$ in which only the lowest level (200 mg ATA $+25 \mathrm{mg} \mathrm{ALA} / \mathrm{kg}$ feed) was tenderer than the control and the other treatments (200 mg ATA +75 or $150 \mathrm{mg}$ ALA $/ \mathrm{kg}$ feed). The higher shear force values in the $160 \mathrm{ppm}$ treatment may have been due to differences in tissue architecture(25).

Addition of ALA reduced cooking loss without affecting WHC and drip loss. With the exception of the $80 \mathrm{ppm}$ treatment, cooking loss was lower in the ALA treatments. No differences $(P>0.05)$ between treatments were observed

Cuadro 5. Efecto de diferentes niveles de ácido alfa lipoico (ALA) en la dieta sobre las características de la carne en pollos de engorda

Table 5. Effect of different $\alpha$-lipoic acid (ALA) inclusion levels on meat quality parameters in broiler chickens

\begin{tabular}{|c|c|c|c|c|c|c|c|c|c|c|c|c|c|c|}
\hline \multirow[t]{2}{*}{$\begin{array}{l}\text { ALA } \\
\text { (ppm) }\end{array}$} & \multicolumn{2}{|c|}{$\begin{array}{l}\text { Shear force } \\
\text { (kgf) }\end{array}$} & \multicolumn{2}{|c|}{$\begin{array}{c}\text { Cooking Loss } \\
(\%)\end{array}$} & \multicolumn{2}{|c|}{$\begin{array}{l}\text { Water holding } \\
\text { capacity (\%) }\end{array}$} & \multicolumn{2}{|c|}{$\begin{array}{c}\text { Drip loss } \\
(\%)\end{array}$} & \multicolumn{2}{|l|}{$\mathrm{pH}_{15}$} & \multicolumn{2}{|l|}{$\mathrm{pH}_{24}$} & \multicolumn{2}{|c|}{$\begin{array}{c}\text { Breast yield } \\
(\%)\end{array}$} \\
\hline & $\bar{x}$ & SE & $\bar{x}$ & SE & $\bar{x}$ & SE & $\bar{X}$ & SE & $\bar{x}$ & SE & $\bar{x}$ & SE & $\bar{x}$ & SE \\
\hline 0 & $1.72 a, b$ & 0.03 & $0.67^{\mathrm{a}}$ & 0.67 & 52.76 & 0.62 & 2.17 & 0.1 & $6.52^{a}$ & 0.04 & $5.25^{\mathrm{a}}$ & 0.0 & 19.21 & 0.04 \\
\hline 40 & $1.59 \mathrm{a}$ & 0.03 & $17.20^{b}$ & 0.67 & 53.94 & 0.62 & 1.99 & 0.1 & $6.65^{b}$ & 0.0 & $5.18^{a}$ & 0.09 & 19.49 & 0.04 \\
\hline 80 & $1.65^{a}$ & 0.03 & $19.28 \mathrm{a}, \mathrm{b}$ & 0.67 & 53.86 & 0.62 & 2.12 & 0.1 & $6.74 \mathrm{~b}$ & 0.04 & $5.05 a, b$ & 0.09 & 19.23 & 0.04 \\
\hline 160 & $1.79^{b}$ & 0.03 & $18.24 \mathrm{~b}$ & 0.67 & 53.42 & 0.62 & 2.17 & 0.1 & $6.73^{b}$ & 0.04 & $4.98^{b}$ & 0.09 & 20.23 & 0.04 \\
\hline 320 & $1.64^{\mathrm{a}}$ & 0.03 & $18.55^{b}$ & 0.67 & 53.90 & 0.62 & 2.06 & 0.1 & $6.60 \mathrm{a}, \mathrm{b}$ & 0.04 & $5.08 \mathrm{a}, \mathrm{b}$ & 0.09 & 19.62 & 0.04 \\
\hline
\end{tabular}

$a, b, c, d$ Different letter superscripts in the same column indicate significant difference $(P<0.05), n=20$.

$\mathrm{SE}=$ Standard error . 
aun cuando no existen reportes en la literatura de mediciones directas sobre la pechuga, sí existen datos de otros autores(21) que evaluaron la carne cruda de la pechuga en presentación de nugget y hamburguesa, y muestran que para el caso de los nuggets el testigo fue similar al tratamiento con la menor dosis (25 mg AAL/ $\mathrm{kg}$ alimento); dicho tratamiento, a su vez, no presentó diferencia con el resto de los niveles de AAL. Mientras que para el caso de la hamburguesa el testigo también fue similar al tratamiento con la menor dosis, pero diferente de las demás concentraciones de $A A L$, todo esto tomando en cuenta únicamente los datos del día 0. Respecto a a* y b*, se puede observar que la carne presentó valores mayores para el índice de amarillo que para el de rojo, esto fue causado, en parte, por un $\mathrm{pH}$ final bajo, que reduce la absorción de la luz verde (valor negativo de a*) de la mioglobina, y resulta en una carne que parece menos roja y más amarilla(23).

En el presente trabajo sólo se evaluó el color de la carne de la pechuga y se debe tomar en cuenta que ésta contiene una baja concentración de mioglobina, por lo que su color es más claro que otras piezas, como el muslo(24). En general, todas las concentraciones de AAL afectaron el color de la carne $(P<0.05)$, los niveles de 40 y 160 ppm de AAL produjeron carne menos luminosa, y con menor intensidad de rojo y de amarillo en comparación con la carne del tratamiento testigo. El nivel de 80 ppm de AAL también mostró carne menos luminosa que el testigo, pero con igual intensidad de color rojo y amarillo que el mismo testigo; a su vez, el tratamiento de 320 ppm AAL solamente disminuyó la intensidad de color amarillo de la carne, pero mostró una luminosidad y una intensidad de color rojo igual que la carne del tratamiento testigo. Sin embargo, para el caso de piel no fue así, pues no se presentó ninguna diferencia atribuida a los tratamientos.

En el Cuadro 5 se observa que la dosis 160 ppm obtuvo el mayor valor en la fuerza de for water holding capacity, drip loss and breast yield. Water holding capacity values aid in quantifying muscle fiber integrity. High WHC values suggest that muscle fiber has maintained its integrity during the transition from muscle to meat. Lower WHC values indicate a greater loss of intracellular liquids from muscle fiber(7). As reported previously(12), drip loss in the tested breast meat did not differ between treatments. Initially developed to evaluate the abundant water loss in pork, this test does not identify notable differences in chicken, which has lower drip-loss values. Extending time period in this test up to three days (72 h) at $4{ }^{\circ} \mathrm{C}$ would better identify any possible differences between treatments(19).

Administering an antioxidant could promote muscle fiber cell membrane integrity, leading to high WHC values $(26,27)$. Only cooking loss values for three of the treatments $(40,160$ and 320 ppm) behaved as expected, while the remaining cooking loss values, and all the WHC and drip loss values did not.

For $\mathrm{pH}_{15}$, the 40,80 and 160 ppm treatments had values different $(P<0.05)$ from the control, while the $320 \mathrm{ppm}$ treatment did not differ $(P>0.05)$ from the control. At $24 \mathrm{~h}\left(\mathrm{pH}_{24}\right)$, the 160 ppm treatment differed $(P<0.05)$ from all the other inclusion levels. Meat color can be influenced by $\mathrm{pH}(28)$; indeed, lower $\mathrm{pH}$ is reported to increase $L^{*}$ values $(28,29,30)$. This agrees with the present initial $\mathrm{pH}$ values $\left(\mathrm{pH}_{15}\right)$ in which the control and 320 ppm treatments had the lowest $\mathrm{pH}$ values and the highest $\mathrm{L}^{*}$ values; these treatments did not differ in $\mathrm{pH}_{15}$ or $L^{*}$.

Both muscle tissue glycogen content and the mechanism that converts glycogen into lactic acid can affect $\mathrm{pH}$ values $(31,32,33)$; these variables were not measured in the present study. Nonetheless, the fact that the 160 ppm $\mathrm{pH}_{24}$ value was lower than the control may be explained by higher muscle glycogen content in this treatment in response to its higher FCR values. Another possible factor affecting muscle 
corte que el resto de los tratamientos $(P>0.05)$, excepto que el tratamiento testigo. Los resultados de fuerza de corte presentan discrepancias con lo observado por otros autores(21), la adición de AAL disminuyó la dureza de la carne con excepción del nivel 160 ppm, el cual mostró una dureza igual que la carne del tratamiento testigo. Cabe mencionar que tales autores(21) evaluaron el efecto a través del tiempo 0, 30, 60 y 90 días, pero si se toma como referencia únicamente el día 0 , entonces el testigo fue igual al resto de las dosis (200 $\mathrm{mg}$ de ATA + 75, $150 \mathrm{mg}$ de AAL/ $\mathrm{kg}$ de alimento), siendo sólo la menor dosis (200 mg de ATA + $25 \mathrm{mg}$ de AAL/kg de alimento) la que fue diferente. Los resultados observados en la fuerza de corte para el tratamiento de $160 \mathrm{ppm}$ pueden ser debidos a cambios en la arquitectura tisular(25).

El AAL disminuyó la pérdida por cocción sin afectar la capacidad de retención de agua y la pérdida por goteo. La pérdida por cocción fue menor en las canales de los animales tratados con AAL, excepto para los animales que recibieron $80 \mathrm{ppm}$. En las pruebas de capacidad de retención de agua, pérdida por goteo y rendimiento de la pechuga no se presentaron diferencias $(P>0.05)$. Los valores de capacidad de retención de agua dan una idea de la integridad de las fibras musculares, pues si éstas conservan una mayor cantidad de agua, se debe a que han mantenido su integridad durante la conversión de músculo a carne y por ende presentarán un valor mayor para esta variable. Contrariamente, al perder demasiada agua el resultado obtenido irá disminuyendo, lo que significa que hay más líquidos intracelulares que han salido de las fibras musculares(7). Tampoco se presentaron diferencias para el caso de la prueba de pérdida por goteo, como otros autores mencionan(12); esta prueba se desarrolló inicialmente para evaluar carne de cerdo en donde las pérdidas de agua son abundantes; sin embargo, para el caso de la carne de pollo que tiende a perder menor cantidad de agua por goteo, sería preferible que la prueba aumentara el periodo de duración glycogen content is pre-slaughter stress in animals.

Overall, $\alpha$-lipoic acid (ALA) had no significant effect on the evaluated productive parameters, although at the $320 \mathrm{ppm}$ inclusion level it decreased feed intake and consequently lowered final weight. Lower levels (40 and 160 ppm ALA) decreased meat luminosity and red color, but neither effect was apparent at the $320 \mathrm{ppm}$ level. Addition of ALA had no effect on skin color. Meat tenderness and cooking loss decreased with ALA inclusion without affecting water holding capacity or drip loss. Initial $\mathrm{pH}$ $\left(\mathrm{pH}_{15}\right)$ was higher with added ALA, but stabilized $24 \mathrm{~h}$ post mortem; the $160 \mathrm{ppm}$ treatment had a slightly more acid pH than the control. Further research is needed using more animals per replicate to determine the optimum ALA level for productive and meat quality parameters.

\section{ACKNOWLEDGEMENTS}

The research was financed by project PAPIITUNAM IN200910. SSG received a CONACYT scholarship to study a Masters in Science at the Facultad de Estudios Superiores-Cuautitlán, UNAM, México.

End of english version

hasta tres días manteniéndose a $4{ }^{\circ} \mathrm{C}$ como lo hicieron otros autores(19), lo que permitiría determinar si realmente se presenta un efecto por causa del tratamiento.

Cabe recordar que al suministrarse un antioxidante a los animales puede promoverse la integridad de la membrana celular de las fibras musculares, por lo cual éstas tendrán mayor capacidad de retener agua $(26,27)$, como se mostró únicamente en la prueba de pérdida de agua por cocción (40, 160 y 320 ppm), un resultado similar era esperado para las pruebas 
de capacidad de retención de agua y pérdida por goteo, pero no fue así.

Los valores obtenidos de la medición del $\mathrm{pH}_{15}$ en los tratamientos 40,80 y 160 ppm fueron diferentes al testigo $(P<0.05)$, aunque la dosis de 320 ppm presentó un resultado similar al resto de las dosis, éste no fue diferente del testigo $(P>0.05)$. Por otro lado, para el caso de la lectura de $\mathrm{pH}_{24}$ sólo el tratamiento de 160 ppm fue diferente del resto de las dosis $(P<0.05)$. Se sabe que el $\mathrm{pH}$ pueden influenciar el color de la carne(28), pues se ha reportado que al disminuir el $\mathrm{pH}$ el valor de L* se incrementa $(28,29,30)$; esto concuerda con los valores de $\mathrm{pH}$ inicial, donde el testigo y el tratamiento $320 \mathrm{ppm}$ tuvieron los valores de pH más bajos y la luminosidad más alta, siendo los valores iguales en ambos, tanto de $\mathrm{pH}_{15}$ como de $L^{*}$.

Dos de los factores que pueden ejercer efecto sobre el valor del $\mathrm{pH}$ son la cantidad de glucógeno contenido en el tejido muscular y el mecanismo que convierte el glucógeno en ácido láctico $(31,32,33)$. Aun cuando en este trabajo no se midieron estas variables, una posible explicación podría ser que la dosis de 160 ppm pudo promover una mayor cantidad de glucógeno muscular por una conversión alimenticia más eficiente, pues obtuvo un valor de $\mathrm{pH}_{24}$ menor que el testigo, aunque no fue diferente de los tratamientos 80 y 320 ppm, pero se sabe que la cantidad de glucógeno disminuye por estrés previo al sacrificio.

En conclusión y a diferencia de trabajos previos, no se observó un efecto de AAL sobre los parámetros productivos; sin embargo, el AAL a dosis elevadas inhibió el consumo en los animales y por ende disminuyó el peso. Los niveles bajos de AAL (40 y 160 ppm) disminuyeron la luminosidad y el color rojo de la carne, pero ese efecto desapareció cuando el nivel de AAL subió a 320 ppm. El AAL no mostró efecto en el color de la piel de las aves. La adición de AAL disminuyó la dureza de la carne y la pérdida por cocción sin afectar la capacidad de retención de agua y la pérdida por goteo. $\mathrm{El} \mathrm{pH}_{15}$ de la carne aumentó con el $\mathrm{AAL}$, pero éste se estabilizó a las $24 \mathrm{~h}$ post mortem, cuando sólo el tratamiento 160 ppm AAL produjo carne ligeramente más ácida que la carne testigo. Estudios adicionales con un mayor número de aves por repetición serán necesarios para determinar cuál es la dosis idónea de AAL que permita obtener los resultados deseados, tanto en granja como en calidad de la carne.

\section{AGRADECIMIENTOS}

Este trabajo fue financiado por el Proyecto PAPIIT-UNAM IN200910. Sergio Sigler Galván agradece la beca otorgada por el CONACYT para la realización de sus estudios de Maestría en la Facultad de Estudios Superiores-Cuautitlán, UNAM, México.

\section{LITERATURA CITADA}

1. SAGARPA. Secretaría de Agricultura, Ganadería, Desarrollo Rural, Pesca y Alimentación. Situación actual y perspectiva de la producción de carne de pollo en México. Coordinación General de Ganadería. México. 2009.

2. Dransfield E, Sosnicki AA. Relationship between muscle growth and poultry meat quality. Poult Sci 1999;78:743746.

3. Nesbitt NM, Cicchillo RM, Lee KH, Grove TL, Booker SJ. Lipoic acid biosynthesis. In: Lipoic acid, energy, production, antioxidant activity and health effects. Patel MS et al editors. First ed. Boca Raton, Florida, USA: CRC Press. Taylor \& Francis Group; 2008:11-13.

4. Shen Q, Jones C, Kalchayanand M, Zhu M, Du M. Effect of dietary alpha-lipoic acid on growth, body composition, muscle $\mathrm{pH}$, and AMP-activated protein kinase phosphorylation in mice. J Anim Sci 2005;83:2611-2617.

5. Wang Y, Li X, Guo Y, Chan L, Guan X. Alpha-lipoic acid increases energy expenditure by enhancing AMPK-PGC-1 $\alpha$ signalling in the skeletal muscle of aged mice. Metabolism 2010;59:967-976.

6. Arshad MS, Anjum FM, Asghar A, Khan MI, Yasin M, Shahid $\mathrm{M}$, El-Ghorab AH. Lipid stability and antioxidant profile of microsomal fraction of broiler meat enriched with $\alpha$-lipoic acid and $\alpha$-tocopherol acetate. J Agric Food Chem 2011;59:7346-7352.

7. Berg E, Maddock K, Linville M. Creatine monohydrate supplemented in swine finishing diets and fresh pork quality: 


\section{EFECTO DEL ÁCIDO LIPOICO SOBRE PARÁMETROS PRODUCTIVOS Y CALIDAD DE LA CANAL EN EL POLLO}

III. Evaluating the cumulative effect of creatine monohydrate and alpha-lipoic acid. J Anim Sci 2003;81:2469-2474.

8. Díaz CA, Serret M, Ramírez G, Ávila E, Guinzberg R, Piña E. Prophylactic action of lipoic acid on oxidative stress and growth performance in broilers at risk of developing ascites syndrome. Avian Pathol 2003;32:645-653.

9. Halici M, Imik $H$, Koç M, Gümüs' R. Effects of a-lipoic acid, vitamins $E$ and $C$ upon the heat stress in Japanese quails. J Anim Physiol Anim Nutr 2012;96:408-415.

10. Hamano Y. Effects of dietary lipoic acid on plasma lipid, in vivo insulin sensitivity, metabolic response to corticosterone and in vitro lipolysis in broiler chickens. Br J Nutr 2006; 95:1094-1101.

11. Le Bihan-Duval E, Debut M, Berri C, Sellier N, SantéLhoutellier V, Jégo $Y$, et al. Chicken meat quality: genetic variability and relationship with growth and muscle characteristics. BMC Genetics 2008;9:53.

12. Petracci M, Baéza E. Harmonization of methodology of assessment of poultry meat quality features. Working paper of WPSA Working Group 5. Poultry Meat. 2009.

13. Le Bihan-Duval $\mathrm{E}$, Millet $\mathrm{N}$, Remignon $\mathrm{H}$. Broiler meat quality: Effect of selection for increased carcass quality and estimates of genetic parameters. Poult Sci 1999;78:822-826.

14. CIE. Commission Internationale de l'Eclairage. 18th session. Londres, Inglaterra. CIE Publication. 1976;36.

15. Alarcón A, Duarte J, Rodríguez F, Janacua H. Incidencia de carne pálida-suave-exudativa (PSE) y oscura-firma-seca (DFD) en cerdos sacrificados en la región del Bajío en México. Téc Pecu Méx 2005;43:335-346.

16. Alarcón A, Gamboa J, Alonso F, Grado J, Janacua H. Efecto de variables críticas del sacrificio sobre las propiedades físico químicas de la carne de cerdo. Téc Pecu Méx 2006;44:53-66.

17. Grau R, Hamm R. Eine einfache Methode zur bestimmung der wasserbindung im muskel. Die Naturwissenschaften 1953;40:29-30.

18. Boakye K, Mittal GS. Changes in $\mathrm{pH}$ and water holding properties of Longissimus dorsi during beef ageing. Meat Sci 1993;34:335-349.

19. Allen $C D$, Fletcher DL, Northcutt JK, Russell SM. The relationship of broiler breast color to meat quality and shelf - life. Poult Sci 1998;77:361-366.

20. Lee WJ, Song KH, Koh EH, Won JC, Kim HS, Park HS, et al. Alpha-lipoic acid increases insulin sensitivity by activating AMPK in skeletal muscle. Biochem Biophys Res Commun 2005;332:885-891.

21. Sohaib M, Anjum F, Khan M, Arshad M, Shahid M. Enhanced of lipid stability of broiler breast meat and meat products fed on alpha lipoic acid and alpha tocopherol acetate supplemented feed. Lipids Health Dis 2012;11:57.

22. Chen $\mathrm{P}, \mathrm{Ma} \mathrm{Q}$, Ji C, Zhang J, Zhao L, Zhang Y, et al. Dietary lipoic acid influences antioxidant capability and oxidative status of broilers. Int J Mol Sci 2011;12:8476-8488.

23. Castellini C, Mugnai C, Dal Bosco A. Effect of organic production system on broiler carcass and meat quality. Meat Sci 2002;60:219-225.

24. Imik H, Ozlu H, Gumus R, Atasever MA, Urcar S, Atasever $M$. Effects of ascorbic acid and a-lipoic acid on performance and meat quality of broilers subjected to heat stress. $\mathrm{Br}$ Poult Sci 2012;53:800-888.

25. MacIntosh BR, Gardiner PF, McComas AJ. Muscle architecture and muscle fiber anatomy. In: Skeletal muscle: Form and function. MacIntosh BR et al editors. 2nd ed. Champaign, Illinois, USA: Human Kinetics; 2006;3-21.

26. Cai S, Wu C, Gong L, Song $T$, Wu H, Zhang L. Effects of nano-selenium on performance, meat quality, immune function, oxidation resistance, and tissue selenium content in broilers. Poult Sci 2012;91:2532-2539.

27. Hu C, Wang D, Pan H, Zheng W, Zuo A, Liu J. Effects of broccoli stem and leaf meal on broiler performance, skin pigmentation, antioxidant function, and meat quality. Poult Sci 2012;91:2229-2234.

28. Schilling $M$, Radhakrishnan $V$, Vizzier-Thaxton $Y$, Chritensen K, Joseph P, Williams J, Schmidt T. The effects of low atmosphere stunning and deboning time on broiler breast meat quality. Poult Sci 2012;91:3214-3222.

29. Pekel A, Demirel G, Midilli M, Yalcintan H, Ekiz B, Alp M. Comparison of broiler meat quality when fed diets supplemented with neutralized sunflower soapstock or soybean oil. Poult Sci 2012;91:2361-2369.

30. Samuel D, Trabelsi S. Influence of color on dielectric properties of marinated poultry breast meat. Poult Sci 2012;91:2011-2016.

31. McKee SR, Sams AR. The effect of seasonal heat stress on rigor development and the incidence of pale, exudative turkey meat. Poult Sci 1997;76:1616-1620.

32. Duclos MJ, Berri C, Le Bihan-Duval E. Muscle growth and meat quality. Poultry meat and egg quality symposium. ] Appl Poult Res 2007;16:107-112.

33. Wang RR, Pan $X J$, Peng ZQ. Effects of heat exposure on muscle oxidation and protein functionalities of pectoralis majors in broilers. Poult Sci 2009;88:1078-1084.

34. Hamano $Y$, Sugawara $S$, Kamota $Y$, Nagai E. Involvement of lipoic acid in plasma metabolites, hepatic oxygen consumption, and metabolic response to a b-agonist in broiler chickens. Br J Nutr 1999;82:497-503. 
Sergio Sigler Galván, et al. / Rev Mex Cienc Pecu 2015;6(2):207-219 